\title{
A step-by-step method for producing 3D crania models from CT data
}

\begin{abstract}
Objectives: Modern computed tomography (CT) databases offer a valuable resource for obtaining skeletal reconstructions and contemporary population data. However, researchers may not utilise CT data due to limited funds for proprietary modelling software, or from a lack of awareness of visualization techniques. This paper presents a step-by-step method for creating accurate 3D crania models from CT data using the free and open-source platform 3D Slicer. This method is tested to 1) establish if novice users can produce 3D crania models following the steps, and 2) determine if these models are accurate to models from experienced users.
\end{abstract}

Materials and Methods: The step-by-step method was recorded and tested by five observers who each produced twenty 3D models using clinical sinus CT scans $(n=20)$. The models $(n=100)$ were evaluated through a quantitative mesh comparison to establish the accuracy with experienced users and against novice users.

Results: The mesh comparison between the models from the experienced observers resulted in an average absolute mean distance of $0.4 \mathrm{~mm}$, with $99 \%$ of models accurate to within $0.5 \mathrm{~mm}$. The novice observers were able create robust 3D models following the stepby-step method with average absolute mean distances of 0.5 to $0.6 \mathrm{~mm}$, and $95 \%$ of the mean distances within $1 \mathrm{~mm}$ of the reference model.

Conclusion: All of the crania models produced were comparably accurate with minor variances seen in the background noise and orbital bone modelling. The tested method is accessible and suitable for use with modern CT databases and for forensic reconstruction approaches.

\section{Keywords}

Forensic anthropology

Virtual anthropology

3D modelling

Computed tomography

Digitisation 


\section{Introduction}

Digitisation techniques are becoming increasingly popular across the archaeological and anthropological sciences for generating models of human osteological specimens [1]. Moreover, modern computed tomography (CT) databases are providing a novel resource for archaeologists and anthropologists to access virtual skeletal data, opening up opportunities for the comparison of modern and historical population data [1] as well as the development of novel techniques for identification [2, 3]. CT scanning is a non-destructive and noninvasive acquisition method traditionally used in medical applications that has distinct advantages in its capabilities for imaging bony structures [4]. However, there can be barriers to the accessibility of CT data including the financial cost of software, a lack of user knowledge and experience with 3D modelling, and no established method. This study presents a step-by-step method that can be used to create 3D osteological models with a free open-source platform and assesses the accuracy of 3D models made through quantitative mesh comparison analysis.

\section{Background}

Virtual anthropology and the application of biological profiling techniques to digital data is well-documented and a growing trend that has facilitated the development of quantitative forensic anthropology methodologies [5]. There is an increasing number of examples of CT scanning being used across archaeology and forensic anthropology that are documented in the published scientific literature for a wide range of sample types and applications [6-13] and CT is increasingly routinely used in forensic institutes for post-mortem examinations [14].

As a result, CT databases are being created using modern populations $[1,15]$, such as the database developed by the Radiology-Pathology Center for Forensic Imaging (CFI) at the University of New Mexico School of Medicine [16], which contains 15,000 whole-body decedent CT scans. CT databases based on post-mortem computed tomography (PMCT) 
data or clinical patient data offer a valuable source of data that better represent modern populations in comparison to historical skeletal collections [1, 17], and can facilitate more opportunities for novel research that will provide new methods of measurement and analysis to ultimately support more robust and valid human identifications.

The virtual modelling process allows for automatic calibration of measurements. This capability enables greater accuracy and quicker data collection of any object or specimen under analysis [18], and so the accuracy and reproducibility of digitisation techniques for obtaining biological profiling data is considered to be greater than traditional linear methods $[5,19]$. The accuracy of a 3D model is fundamentally linked to the scanning and modelling parameters employed [4, 20-22]. Moreover, while CT imaging parameters are vital for achieving high quality data $[14,21,23]$, it is not necessary for end-users (e.g. archaeologists or anthropologists) to have expertise in generating CT scans; a skilled radiographer will image a sample based on its properties (e.g. if it is wet, dry, soft, hard or containing metal) and the final intended use of the data (which informs the level of detail required) [4]. The end-user can then use the CT data to produce 3D models.

\section{D modelling Methods}

Best practice guidelines for surface scanning [24], a CT dataset for biological profiling in forensic anthropology [25], and useful 3D printing primers tailored for radiologists [26, 27] are available. Additionally, online good practice guides [28], are also easily accessible which include notes on laser scanning, photogrammetry as well as data collection, analysis and visualisation. However, there is currently a lack of established methods available for producing 3D models from CT data in forensic anthropology that are specifically aimed at non-CT experts.

To produce a CT scan, a beam of ionising radiation is passed through a sample to create an image in slices that can then be digitally recorded as Digital Imaging and Communications in 
Medicine (DICOM) data. Medical imaging data (including DICOM data) can be viewed in two-dimensions (2D) as a multiplanar reconstruction (MPR) in coronal, axial and sagittal views. Additionally, the stack of volumetric data (slices) can be rendered as a threedimensional (3D) surface volume rendering [19]. Areas of differing densities, such as skin, tissue, bone or metal can automatically be differentiated and rendered individually due to differences in x-ray attenuation [4]. A 3D volume rendering can be automatically generated using DICOM viewing software and 2D images (screenshots) or 3D animations (videos), however this rendering is not exportable and therefore not printable.

In order to produce an exportable file-type the DICOM data must be segmented, a process that requires a user to manually carry out a series of steps. Segmentation exploits the difference in material density (measured in Hounsfield units) to distinguish between regions of interest (ROI). Segmentation can be achieved using thresholding, a method that applies a label map to a ROI, through segmenting objects based on their density (measured in Hounsfield units). Subsequently, a modelling algorithm can convert the labelled DICOM data into an exportable surface model, commonly an STL (Stereolithography) file format. DICOM data rendering and segmentation is performed using a DICOM viewer software platform. There are a number of different DICOM viewers available, see Hodgdon, et al. [26] for examples of software for creating 3D prints from CT data. With so many different software packages available, scientific studies are inevitably based on a variety of software. This has led to inconsistency within the field, and results can rarely be directly compared between studies whereby different software and methodologies have been implemented.

Additionally, a major limitation to many proprietary software packages is the associated financial cost. The use of free software may be more fit-for-purpose in forensic science where practitioners and researchers often do not have the budget for expensive licence fees. 3D Slicer [29] is a CT reconstruction software (DICOM viewer) that was developed with support from the US National Institutes of Health [30] for surgical planning and visualisation 
of medical imaging data. 3D Slicer was specifically designed as a research tool and as such it is highly accessible; it is free, open-source, cross-platform, supports extensions and has a non-restrictive license [29] making it a potentially valuable tool for forensic anthropology and identification approaches.

Presently, many specialists working with human remains have not received training in or been exposed to 3D modelling. As a result, it has been recommended that anthropologists be trained in 3D modelling and digital data interpretation to develop modern forensic anthropology methodologies [31]. Given the advantages of working with CT databases and the superior analytical prospects of virtual skeletal models, the ability to handle and utilise CT data is an emerging challenge facing researchers. The purpose of this paper was to enhance the ability of a user to handle CT data for the examination of human remains. This was addressed by creating an accessible and tested method that users (such as archaeologists or forensic anthropologists) with limited experience of 3D modelling CT data can easily use to create virtual skeletal models to assist with forensic identification.

\section{Material and Methods}

\section{D Slicer Guidelines}

A step-by-step method was designed for application with 3D Slicer to create 3D models of bones from CT data (Figure 1). The method was designed with 3D Slicer version 4.9.0 for Mac (3D Slicer, Brigham Women's Hospital, Boston, MA, US), which can be downloaded from https://www.slicer.org/; for support with installation and set-up of 3D Slicer (see https://www.slicer.org/wiki/Main_Page) [29]. This step-by-step method records each step in the general 3D modelling process using CT scan data and were divided into seven distinct stages:

1. Importing

2. Creating a volume render

3. Manoeuvring model 
4. Cropping volume

5. Segment data using Thresholding effect;

6. Make model

7. Export model as STL.

Each stage incorporated details of which tools to use and were specific to modelling bone tissue (see Figure 1). The step-by-step method was then presented to novice observers to establish if they were able to produce 3D models, and to determine if the models were comparably accurate to the experienced users. 
1. Importing

- Import DICOM data into 3D Slicer

- Drag and drop the file/folder in the 3D Slicer window

'Select a Reader' pop-up window: Click OK

- 'Directory import completed' pop-up window: Click OK

- Load DICOM data using 'Welcome to Slicer module'

- Navigate modules using dropdown menu (see diagram for 3D Slicer interface)

- Volume opens in DICOM module

- Adjust viewport using button if needed (see diagram for 3D Slicer interface)

- Use 'Conventional' for segmenting

2. Creating a volume render

- Volume Rendering Module - select 'Volume' to view from drop down menu

NB. Smaller slice thickness and higher filter values for better resolution of bone

- Toggle eye-shapped button to turn volume render on/off in display

- Select a suitable 'Display' from 'Preset' dropdown menu (hover mouse over different displays to see name)

- Presets for viewing bone: 'CT Bones' or 'CT-AAA2'

- Select button to 'Centre the 3D view on the scene' (see diagram for 3D Slicer interface)

- Slide 'Display - Shift' bar to adjust threshold level or render if needed

3. Manoeuvring model

- Use trackpad/mouse [click and drag] to rotate model in space

- Zoom model in and out using mouse/trackpad

- Translate model's position [shift + click and drag] to move model in space

- Use pin button to change view (left; right; inferior; superior; anterior; posterior) (see diagram for 3D Slicer interface)

4. Cropping volume

- Volume rendering module

- Enable volume cropping

Under 'Crop', turn 'Enable' on: tick tickbox

- Enable ROI view to be on

- Toggle eye button under 'Crop' 'Display ROI'

- Adjust ROI to desired region, use all four viewports (2D and 3D)

- Go to Crop Volume module

- Under 'IO' choose 'Input volume' (should be correct)

Output volume: create new volume (will appear with 'cropped' on end of original volume name)

Select 'Apply'

- Volume Rendering module

- Turn 'Display ROI' off (toggle eye button) and disenable cropping (tickbox)

- Select new cropped volume to view, adjust pre-set so can view bones

- Turn volume render view off (toggle eye button)

5. Segment data using Thresholding effect

- Editor module

- Master volume: select original volume or new cropped volume

- Popup window asking to select 'Generic Anatomy Colors' label map: click OK

- Under 'Edit Selected Label Map' select tool 'ThresholdEffect'

- Select a thresholding label to use (this applies colour/name to volume data)

- E.g. label 2 uses a yellow colour called 'bone'

- Adjust 'Threshold Range' using slider bar to ensure bone is labelled (coloured in), scroll

through $\mathrm{R}, \mathrm{Y}, \mathrm{G}$ views

NB. You can hover mouse over a ROI to see its value (Hounsfield unit, HU) under 'Data

Probe' section

NB. By ticking 'Show Zoomed Slice' you can visualise ROI zoomed in for more detail

NB. Lower value for thresholding bone is generally around 100-300 HU

- Click 'Apply' (ROI in filled in with label)

6. Make model

- Editor module

- Under 'Edit Selected Label Map' select tool 'Make Model Effect'

- Ensure the correct Label is selected (e.g. 'bone')

Alter name of model if needed

- Click 'Apply'

-3D model will appear in 3D viewport (click button to 'Centre the 3D view on the scene' if not)

- Check quality of modelling. If model is over/under labelled, undo and repeat step 5, adjusting

the threshold value as needed

- Access models in 'Model Maker' module

. Export model as STL

- Click 'Save' button (see diagram for 3D Slicer interface) to view 'Save Data' window

- 3D model appears as a Poly Data (.vtk) file

- Change file format to STL (.stl) and change directory to desired location

- Click 'Save'

Figure $13 D$ Slicer step-by-step method 
The step-by-step method was updated following feedback from the initial observers to include a glossary of terms (Table 1) and an annotated screenshot of the 3D Slicer interface (Figure 2) to assist users unfamiliar with the interface. Technical terms relating to CT data and segmentation from within the guidelines were incorporated into the glossary and each term explained. For the 3D Slicer interface annotated screenshot, an image was taken from the 'Welcome to Slicer' module when no data was present. The screenshot was annotated to display the three main aspects of the interface (the toolbar, the module menu and the viewport) and a selection of key buttons that feature in the guidelines. Additionally, the steps were updated to more clearly describe each stage, including the addition of symbols and references to the interface annotated screenshot.

Table 1 Glossary of terms to complement guidelines

\begin{tabular}{ll}
\hline Term & Description \\
\hline CT & Computed Tomography (a radiographic imaging technique) \\
DICOM & A file format for medical image data, including CT volume data \\
Volume & CT data reconstructed in 3D from 2D images \\
Volume render & Surface model on screen (non-exportable) \\
Thresholding & Method used to segment an object based on its density (measured in Hounsfield Units) \\
Volume cropping & Isolating a region (volume) of interest (ROI) \\
ROI & Region of Interest \\
Segmentation & Defining boundaries to a ROI to enable modelling \\
STL & Stereolithic; a file format for 3D data \\
Modules & Platforms for different interfaces to perform different functions \\
\hline
\end{tabular}




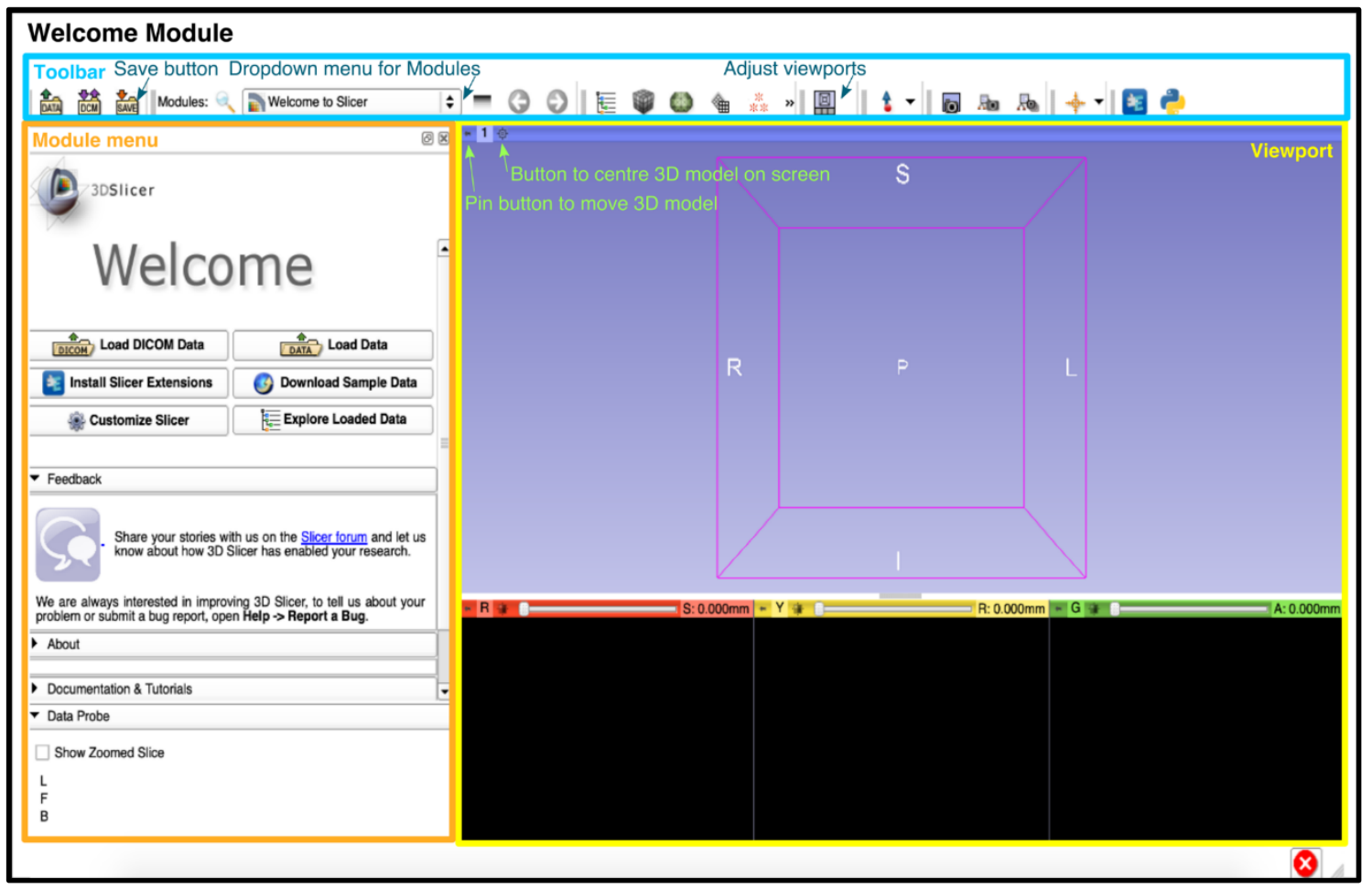

Figure 2 Annotated 3D Slicer interface screenshot

\section{Method Testing Protocol}

Twenty clinical sinus CT scans (ten female and ten male) were obtained from a wider database of $500 \mathrm{CT}$ scans provided by the picture archiving and communications (PACS) department at University College London Hospital from the hospital-wide database. Scans were anonymised but contained minimal demographic data. Approval was received from the Health Research Authority (HRA) and deemed exempt from requiring NHS REC approval by the HRA. The age of the patients ranged from 29-64 years for females and 26-91 years for males. All scans were acquired using $100 \mathrm{kVp}$ at $1 \mathrm{~mm}$ slice thickness on three different scanners with variable reconstruction diameters, currents and filters (see Table 2); 16 of the 20 scans contained flaring artefacts from metallic dental fillings [32].

\section{Table 2 CT scan parameters}

\begin{tabular}{llllll}
\hline Crania & CT Manufacturer & $\begin{array}{l}\text { Reconstruction } \\
\text { diameter }(\mathbf{m m})\end{array}$ & $\begin{array}{l}\text { Tube current } \\
\text { (mA) }\end{array}$ & $\begin{array}{l}\text { Reconstruction } \\
\text { algorithm/filter }\end{array}$ & $\begin{array}{l}\text { Spiral Pitch } \\
\text { Factor }\end{array}$ \\
\hline $\mathbf{1}$ & Siemens Somatom Definition AS+ & 259 & 26 & $\mathrm{H}$ & 0.8 \\
$\mathbf{2}$ & Siemens Somatom Definition AS+ & 258 & 27 & $\mathrm{H} 60 \mathrm{f}$ & 0.8 \\
$\mathbf{3}$ & Siemens Somatom Definition AS+ & 237 & 26 & $\mathrm{Q} 40 \mathrm{f}$ & 0.8 \\
$\mathbf{4}$ & Siemens Somatom Definition AS+ & 169 & 26 & $\mathrm{Q} 40 \mathrm{f}$ & 0.8 \\
$\mathbf{5}$ & Siemens Somatom Definition AS+ & 255 & 31 & $\mathrm{Q} 40 \mathrm{f}$ & 0.8 \\
$\mathbf{6}$ & Siemens Sensation 64 & 223 & 72 & H60f & 0.9 \\
\hline
\end{tabular}




\begin{tabular}{|c|c|c|c|c|c|}
\hline 7 & Siemens Sensation 64 & 154 & 72 & H60f & 0.9 \\
\hline 8 & Siemens Somatom Definition AS+ & 205 & 93 & Q40f & 0.8 \\
\hline 9 & Siemens Somatom Definition AS+ & 277 & 29 & Q40f & 0.8 \\
\hline 10 & Siemens Somatom Definition AS+ & 147 & 20 & J70h & 0.8 \\
\hline 11 & Toshiba Aquilion One & 202.2 & 80 & FC08 & - \\
\hline 12 & Siemens Sensation 64 & 188 & 72 & $\mathrm{H} 60 \mathrm{f}$ & 0.9 \\
\hline 13 & Siemens Somatom Definition AS+ & 195 & 43 & $\mathrm{H} 60 \mathrm{~s}$ & 0.8 \\
\hline 14 & Siemens Somatom Definition AS+ & 195 & 28 & H60f & 0.8 \\
\hline 15 & Siemens Somatom Definition AS+ & 228 & 27 & Q40f & 0.8 \\
\hline 16 & Siemens Somatom Definition AS+ & 204 & 28 & H60f & 0.8 \\
\hline 17 & Siemens Somatom Definition AS+ & 234 & 25 & H60f & 0.8 \\
\hline 18 & Siemens Somatom Definition AS+ & 186 & 30 & Q40f & 0.8 \\
\hline 19 & Siemens Somatom Definition AS+ & 234 & 25 & Q40f & 0.8 \\
\hline 20 & Siemens Somatom Definition AS+ & 214 & 31 & H60f & 0.8 \\
\hline
\end{tabular}

Three observers (observers 1-3) produced twenty separate 3D crania models as exportable STL files using 3D Slicer following the step-by-step method. The method was then updated to accommodate recommendations for improved ease-of-use. Two additional observers (observers 4 and 5) subsequently then created the 3D models using the updated guidelines. Observers 1 and 2 each had c.3 years of experience of working with 3D Slicer, CT data and 3D modelling. Observers 3-5 had no previous experience in using 3D Slicer, CT data or 3D modelling. Additionally, observer 5 was not trained in osteology while the other observers were. Each observer completed data collection on the computer of observer 1 with the software already installed. Models were generated on separate occasions with modelling taking approximately 1 hour for observers 1 and 2, and approximately 4-6 hours for the other observers split over one or two days.

Quantitative analysis was performed via a mesh comparison method using CloudCompare (Version 2.10.2 for Mac, available from https://asmaloney.com/software/), a free, opensource software for comparing point clouds and 3D mesh models. The cloud-to-mesh distance tool [33] was employed to measure the difference between the 3D crania models, which provides a value for the mean distance with a standard deviation (SD), as well as colour scalar maps illustrating the differences (as in Villa, et al. [34]). The crania models made by the two experienced users (observer 1 and 2 ) were initially compared to generate a criterion for the accuracy comparison and determine a 'reference model', after which the remaining crania models from the novice observers 3-5 were compared to that accuracy 
value and 'reference model'. An accuracy threshold of $\pm 1 \mathrm{~mm}$ was also incorporated (as specified as ideal for osteometric data by Carew, et al. [35]).

The mesh comparison data was analysed in Microsoft Excel version 16.36 for Mac (Microsoft, Redmond, WA, US). To assess the inter-observer distances values, withinsubject standard deviations (wSD) and 95\% repeatability were calculated (as in [22, 36, 37]).

\section{Results}

The CloudCompare mesh comparison data for all observers are provided in Table 3. The mesh comparison between the experienced users (observer 2 and observer 1 as the reference model) resulted in mean distances ranging from -0.5 to $1.9 \mathrm{~mm}$, with an overall mean value of $0.1 \pm 0.9 \mathrm{~mm}$ and an average absolute mean of $0.4 \mathrm{~mm}$. Model 14 resulted in a higher mesh distance with a mean of $1.9 \mathrm{~mm} \pm 4.2 \mathrm{~mm}$. Aside from this anomalous result all mean mesh distances were within $\pm 0.5 \mathrm{~mm}$ for observer 2 (to one significant figure), thus this value was used as the accuracy threshold for the novice mesh comparison data. $99 \%$ of the models produced by the experienced observers were accurate to within $0.5 \mathrm{~mm}$.

The mesh comparison between the novice users and the reference models (chosen to be observer 1), ranged from -1.1 to $5 \mathrm{~mm}$, with average absolute means per observer of 0.5 to $0.6 \mathrm{~mm} .74 \%$ of all novice models were within $\pm 0.5 \mathrm{~mm}$, and $95 \%$ were accurate to within 1 $\mathrm{mm}$. Observer 3 produced four models with mean distances greater than $\pm 0.5 \mathrm{~mm}$ (see * in Table 3). Observers 4 and 5 both produced eight models each that were greater than \pm 0.5 $\mathrm{mm}$. Model 5 had particularly greater mesh distances for novice observers 3 and 5 , the scalar map in Figure 3 demonstrates that this difference is due to excess noise modelled within the cranium. 

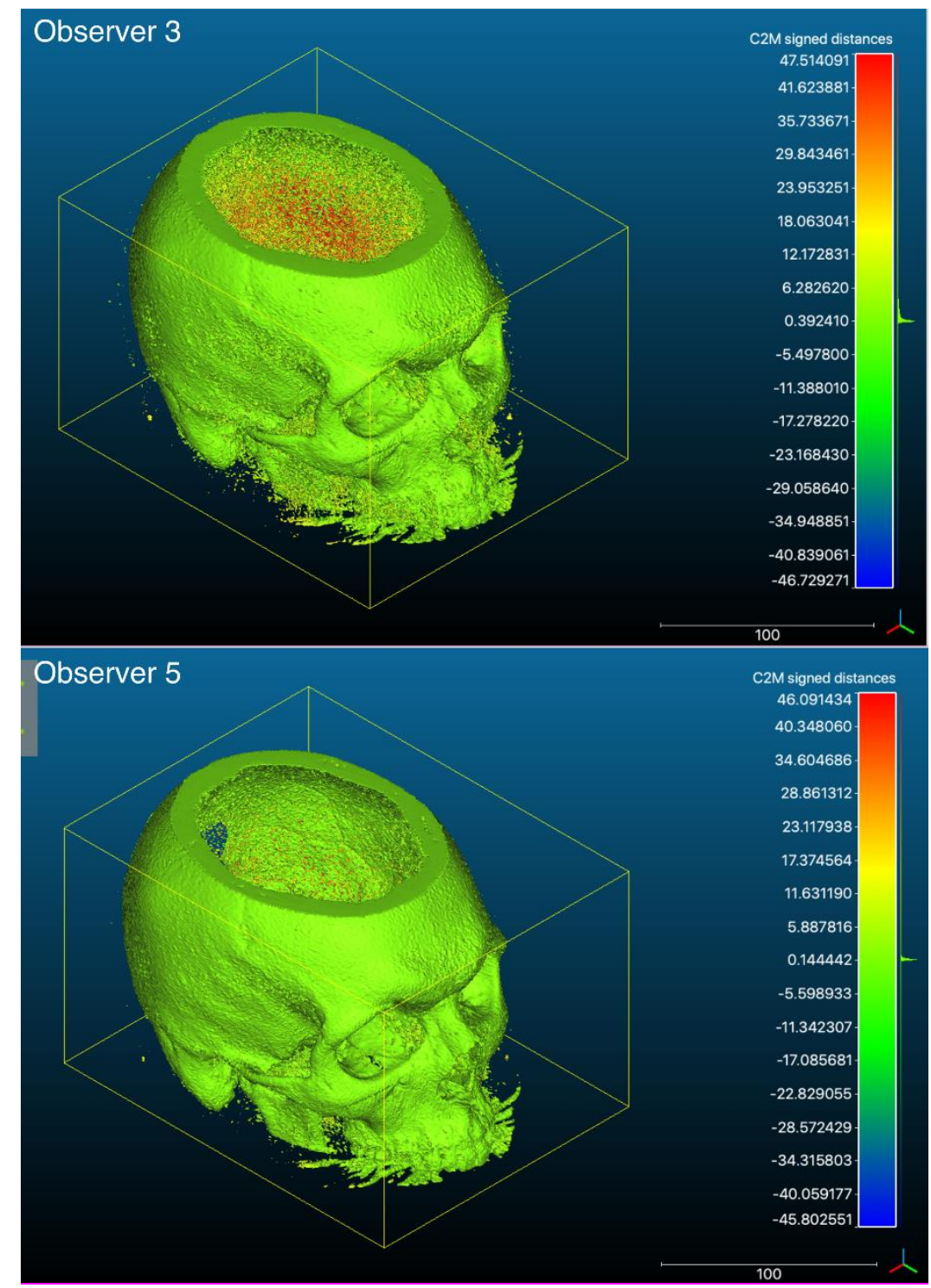

Figure 3 Colour scale illustrating mesh distances between observers 3 and 5 and the reference model for crania model 5 
Table 3 Mesh comparison data for the distance $( \pm S D$, standard deviation) between the reference models (observer 1) and observers 2-5 (mm). Lower section: average data per observer, absolute mean distance, withinsubject standard deviation (WSD) and 95\% repeatability. Righthand section: average data per model. * $=$ mean distance values $> \pm 0.5$

\begin{tabular}{|c|c|c|c|c|c|c|c|c|c|c|}
\hline \multirow[b]{2}{*}{ Model Number } & \multicolumn{2}{|c|}{$\begin{array}{c}\text { Observer } 2 \\
\text { (experienced) }\end{array}$} & \multicolumn{2}{|c|}{$\begin{array}{c}\text { Observer } 3 \\
\text { (novice) }\end{array}$} & \multicolumn{2}{|c|}{$\begin{array}{c}\text { Observer } 4 \\
\text { (novice) }\end{array}$} & \multicolumn{2}{|c|}{$\begin{array}{c}\text { Observer } 5 \\
\text { (novice) }\end{array}$} & \multirow[t]{2}{*}{$\begin{array}{c}\text { Average } \\
\text { mean }\end{array}$} & \multirow[t]{2}{*}{$\begin{array}{c}\text { Average } \\
\text { SD }\end{array}$} \\
\hline & Mean & $S D$ & Mean & $S D$ & Mean & $S D$ & Mean & $S D$ & & \\
\hline 1 & -0.2 & 0.8 & -0.2 & 0.3 & -0.1 & 0.2 & -0.1 & 0.1 & -0.1 & 0.3 \\
\hline 2 & 0.5 & 0.9 & 0.1 & 0.3 & -0.4 & 0.4 & -0.1 & 0.1 & 0.0 & 0.4 \\
\hline 3 & -0.4 & 0.4 & $-0.6^{*}$ & 0.6 & $-0.6^{*}$ & 0.6 & -0.3 & 0.4 & -0.5 & 0.5 \\
\hline 4 & -0.4 & 0.6 & $2.1^{*}$ & 2.5 & 0.5 & 0.7 & $1.1^{*}$ & 1.8 & 0.8 & 1.4 \\
\hline 5 & -0.3 & 0.4 & $5.0^{*}$ & 7.2 & $-0.7^{*}$ & 0.6 & $1.9^{*}$ & 4.6 & 1.5 & 3.2 \\
\hline 6 & 0.3 & 1.2 & 0.4 & 1.2 & $0.9^{*}$ & 2.4 & 0.1 & 0.4 & 0.4 & 1.3 \\
\hline 7 & 0.2 & 1.1 & 0.2 & 1.2 & $0.8^{*}$ & 2.2 & -0.1 & 0.2 & 0.3 & 1.2 \\
\hline 8 & -0.5 & 0.7 & 0.1 & 0.6 & -0.3 & 0.6 & -0.2 & 0.2 & -0.2 & 0.5 \\
\hline 9 & -0.4 & 0.6 & 0.3 & 0.7 & $-1.1^{*}$ & 0.8 & 0.0 & 0.4 & -0.3 & 0.6 \\
\hline 10 & 0.2 & 1.1 & 0.4 & 1.3 & 0.0 & 0.3 & $0.9^{*}$ & 2.3 & 0.4 & 1.2 \\
\hline 11 & 0.5 & 0.4 & $1.4^{*}$ & 2.9 & -0.1 & 0.1 & 0.1 & 0.1 & 0.5 & 0.9 \\
\hline 12 & 0.4 & 1.9 & 0.1 & 2.6 & -0.1 & 0.3 & $1.5^{\star}$ & 2.6 & 0.5 & 1.9 \\
\hline 13 & -0.1 & 0.2 & 0.4 & 0.9 & -0.3 & 0.3 & $0.8^{*}$ & 1.1 & 0.2 & 0.6 \\
\hline 14 & $1.9^{*}$ & 4.2 & -0.3 & 0.3 & -0.1 & 0.5 & $0.7^{*}$ & 2.1 & 0.5 & 1.8 \\
\hline 15 & -0.5 & 0.6 & -0.1 & 0.1 & $-0.9^{*}$ & 0.7 & 0.3 & 1.0 & -0.3 & 0.6 \\
\hline 16 & 0.0 & 0.1 & -0.1 & 0.2 & -0.2 & 0.2 & 0.0 & 0.2 & -0.1 & 0.2 \\
\hline 17 & 0.5 & 1.3 & 0.1 & 0.5 & -0.3 & 0.4 & $0.8^{*}$ & 0.6 & 0.3 & 0.7 \\
\hline 18 & -0.3 & 0.4 & -0.2 & 0.3 & $-0.6^{*}$ & 0.5 & 0.1 & 0.6 & -0.2 & 0.4 \\
\hline 19 & 0.0 & 0.2 & -0.1 & 0.2 & $-1.0^{*}$ & 0.6 & 0.2 & 0.4 & -0.3 & 0.3 \\
\hline 20 & 0.5 & 1.4 & -0.2 & 0.2 & -0.3 & 0.3 & $1.1^{*}$ & 1.8 & 0.3 & 0.9 \\
\hline Average mean & 0.1 & 0.9 & 0.4 & 1.2 & -0.2 & 0.6 & 0.4 & 1.1 & & \\
\hline Absolute mean & 0.4 & & 0.6 & & 0.5 & & 0.5 & & & \\
\hline$w S D$ & 0.6 & & 0.8 & & 0.7 & & 0.7 & & & \\
\hline 95\% Repeatability & 1.8 & & 2.2 & & 1.9 & & 2.0 & & & \\
\hline
\end{tabular}

The within-subject standard deviation (wSD) and 95\% repeatability results did not show any obvious difference between the novice users and experienced user data. Overall, wSD ranged from 0.6 to $0.8 \mathrm{~mm}$ with $95 \%$ repeatability ranging from 1.8 to $2.2 \mathrm{~mm}$.

A visual comparison of the crania models from each observer showed no major differences to the macromorphology of the crania between-observers. Differences were seen in the amount of background noise included and in the variation of the amount of bone surface modelled in the orbits between observer 2 (experienced) and the novice observers (Figure 4). 


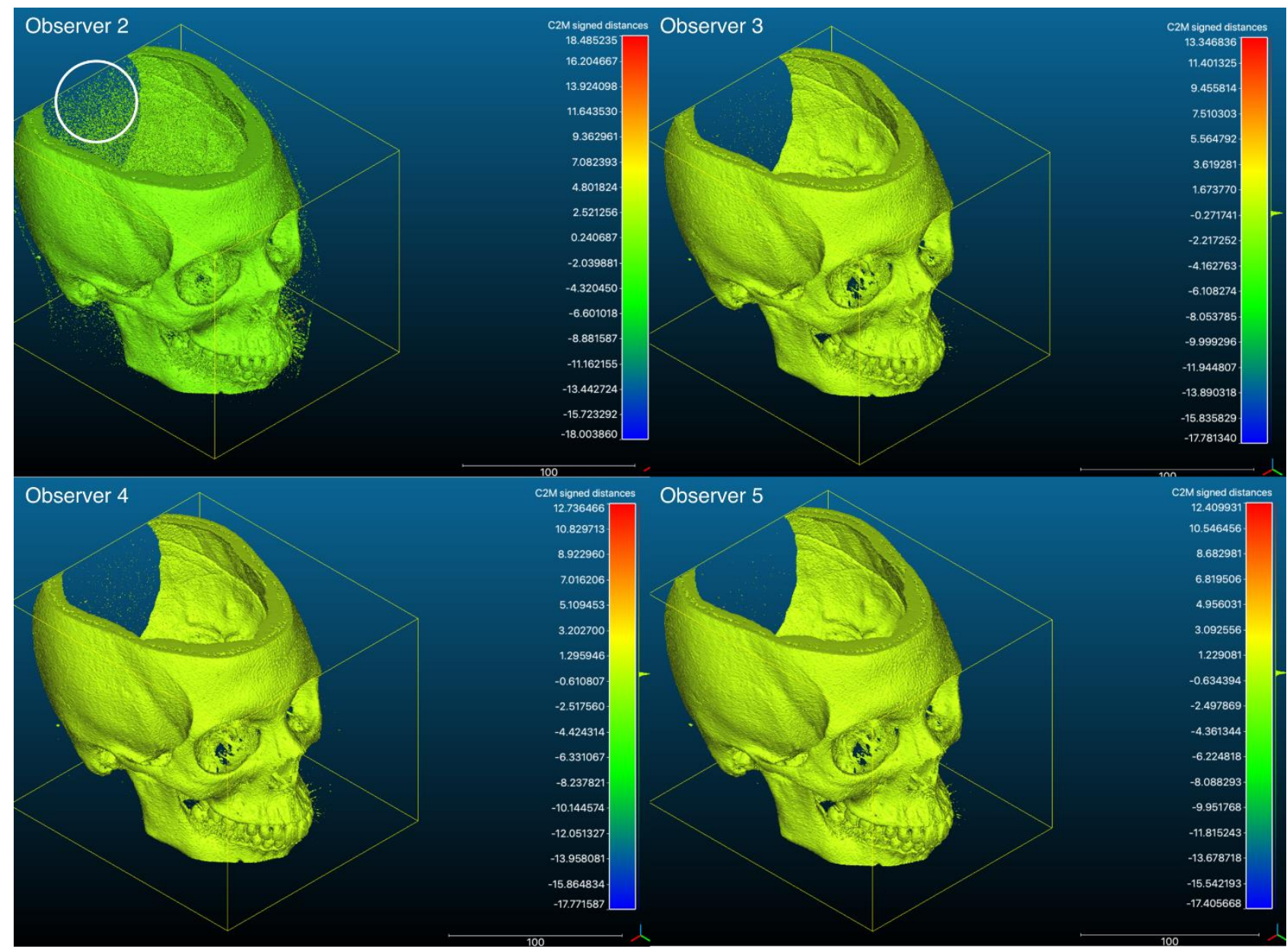

Figure 4 Colour scalar maps of crania model 1 (compared to the reference model) from observers 2-5.

Differences can be seen with background noise (white circle) and in the orbital bone modelling

\section{Discussion}

An easy-to-use method for modelling CT data has the potential to be an invaluable tool that can be utilised by non-experts to generate good-level virtual skeletal models. This experiment provides empirical data testing the utility of the step-by-step method developed for this study. The quantitative mesh comparison showed that the experienced users produced models of comparable dimensions and that the majority of the models from the novice users $(75 \%)$ were accurate to the reference model to within $\pm 0.5 \mathrm{~mm}$, and $95 \%$ were accurate to within $\pm 1 \mathrm{~mm}$. This accuracy threshold was additionally the same value as the CT slice thickness $(1 \mathrm{~mm})$, which is intrinsically linked to the level of accuracy obtainable with these 3D models (as noted in the background section). Many of the differences seen 
may be due to the background noise that was included in the mesh comparison, this noise could easily be deleted using a range of visualisation software programs and may result in even lower mesh distance values, however this was outside the scope of this study. The inter-observer distances resulted in low within-subject standard deviations and similar repeatability, indicating that there was little difference between the experienced user and the novice users modelling overall.

The color scalar maps showed that the 3D crania models made by each observer were of good overall quality, with no major macromorphological differences observed beyond the variation of the orbital bones and the amount of background noise generated. This variation by the experienced user (observer 2) is due to a different approach in thresholding wherein the user filled in the orbital bones knowing that the excess noise could be deleted in postprocessing. Conversely, it appears the novice users tried to reduce the amount of background noise in the initial thresholding step, which resulted in more holes in the orbital bones. The novice users were unaware of post-processing that can clean the models. This is typical of observer variation seen in a method that is dependent upon the tacit knowledge of the user.

Observer 3 (with no previous modelling experience) found difficulty with understanding certain terms used in the step-by-step method. The method was then updated to address this, and consequently observers 4 and 5 with no previous modelling experience were able to successfully create models of the crania following the steps. Although the modelling time of each observer was not recorded, it was observed that they generally became faster at modelling with increased practice, i.e. as they became familiar with the steps within the method. Ultimately, the results of the mesh-to-mesh comparison indicate the suitability of this step-by-step method to produce 3D models from CT scans for novice users. 
The crania scans selected were modern clinical scans that did not exhibit any obvious pathology or trauma. In this paper it is noted that the crania scans did not include the whole crania. Although minor surface differences were seen in the orbital bone and background noise between the models, this was due to the segmentation process which could have been affected by the dental flaring. Metallic dental amalgams are known to affect CT scans, ${ }^{33}$ and are going to be a common occurrence when using modern CT data. Nevertheless, the major crania landmarks are not adjacent to the dentition and the overall morphology of the crania appears to have been unaffected by the flaring. With more experience a user will become more knowledgeable in thresholding and could be expected to produce higher quality models, as seen with the experienced observers. Furthermore, the quality of the models produced could have been improved with post-processing to remove background artefacts.

As the data used in this study was derived from living patients there were many ethical considerations that needed to be taken into account. For example, the DICOM data was anonymised prior to collection by the PACS department, the data had to be securely stored and could not be used on computers other than that of the principal researcher. While clinical CT databases offer a valuable resource to obtain modern population data, there are additional ethical concerns and authorisations (such as anonymising clinical data and data protection) that need to be considered prior to data collection.

In this study, five observers were tested in the task of creating 3D models using the step-bystep method. The success of each observer demonstrates that this method can be followed by users with little-to-no previous 3D modelling experiences. Overall, these findings indicate that the step-by-step method offers a potentially valuable tool to enable the production of $3 D$ models from CT data that is free of financial cost and requires no previous experience. Further work to verify the step-by-step method using different CT datasets, and utilising observers from different backgrounds will be valuable for enhancing the accessibility of the method allowing applications more broadly. While this step-by-step method was designed for 
use with skeletal features, it could potentially be applied to other materials such as soft tissues or ceramics as well.

Additionally, whilst resources are available on the internet to support users in self-learning software, these resources have not been empirically tested or validated for specific applications. This method has shown to be useful for training novice users in 3D modelling of CT data, and could be a valuable teaching tool in forensic anthropology training to help ensure the next generation of forensic anthropologists become both skilled in virtual anthropology methods and become capable of taking advantage of modern CT databases. Although users must be vigilant in addressing the higher ethical considerations involved when accessing clinical data.

\section{Conclusion}

This study developed a method for application in archaeology and forensic anthropology to assist 3D modelling CT data and broaden access to this capability. The method enabled:

1) Novice users to create $3 D$ models following the step-by-step method.

2) The production of robust and accurate 3D models of human crania.

The step-by-step method presented here offers a tested tool for creating 3D virtual models of CT data using 3D Slicer, which can be implemented by those with little modelling experience. It is appropriate for forensic anthropology contexts were funding may be restricted and was designed to create a pathway to access new areas of research that can be derived from modern CT data and to assist in individual identification and forensic reconstruction approaches. This approach can further be updated as new versions of 3D slicer are released, and as more models are created.

\section{Acknowledgements}

The authors would like to extend their gratitude to each of the observers that volunteered their time to take part in this research and to the picture archiving and communications 
department at University College London Hospital for providing the CT scan data. We would also like to thank the anonymous reviewers for their time and constructive feedback.

\section{Funding}

This research did not receive any specific grant from funding agencies in the public, commercial, or not-for-profit sectors.

\section{References}

1. Villa C, Buckberry J, Lynnerup N. Evaluating osteological ageing from digital data. J Anat. 2019;235(2):386-95. DOI: 10.1111/joa.12544.

2. Simmons-Ehrhardt TL, Ehrhardt CJ, Monson KL. Evaluation of the suitability of cranial measurements obtained from surface-rendered CT scans of living people for estimating sex and ancestry. J Forensic Radiol Imaging. 2019;19:100338. DOI: 10.1016/j.jofri.2019.100338.

3. Robles M, Morgan RM, Rando C. A novel method for producing 3D models of paranasal sinuses for forensic anthropology applications. Aust J Forensic Sci. 2020:1-10. DOI: $10.1080 / 00450618.2020 .1766113$.

4. $\quad$ Carew RM, Errickson D. Imaging in Forensic Science: Five Years On. J Forensic Radiol Imaging. 2019;16:24-33. DOI: 10.1016/j.jofri.2019.01.002.

5. Decker SJ, Davy-Jow SL, Ford JM, Hilbelink DR. Virtual determination of sex: metric and nonmetric traits of the adult pelvis from 3D computed tomography models. J Forensic Sci. 2011;56(5):1107-14. DOI: 10.1111/j.1556-4029.2011.01803.x.

6. McKnight LM, Adams JE, Chamberlain A, Atherton-Woolham SD, Bibb R. Application of clinical imaging and 3D printing to the identification of anomalies in an ancient Egyptian animal mummy. J Archaeol Sci Rep. 2015;3:328-32. DOI: 10.1016/j.jasrep.2015.06.028. 7. Gill-Robinson H, Elias J, Bender F, Allard TT, Hoppa RD. Using image analysis software to create a physical skull model for the facial reconstruction of a wrapped akhmimic mummy. Journal of Computing and Information Technology CIT. 2006;14(1):45-51. DOI: 8. Benazzi S, Bertelli P, Lippi B, Bedini E, Caudana R, Gruppioni G, et al. Virtual anthropology and forensic arts: the facial reconstruction of Ferrante Gonzaga. J Archaeol Sci. 2010;37(7):1572-8. DOI: 10.1016/j.jas.2010.01.018.

9. Fantini M, de Crescenzio F, Persiani F, Benazzi S, Gruppioni G. 3D restitution, restoration and prototyping of a medieval damaged skull. Rapid Prototyp J. 2008;14(5):31824. DOI: $10.1108 / 13552540810907992$.

10. Kappelman J, Ketcham RA, Pearce S, Todd L, Akins W, Colbert MW, et al. Perimortem fractures in Lucy suggest mortality from fall out of tall tree. Nature. 2016;537(7621):503-7. DOI: 10.1038/nature19332.

11. Micciche R, Carotenuto G, Sineo L. The utility of 3D medical imaging techniques for obtaining a reliable differential diagnosis of metastatic cancer in an Iron Age skull. Int $\mathrm{J}$ Paleopathol. 2018;21:41-6. DOI: 10.1016/j.ijpp.2017.03.006.

12. Miles J, Mavrogordato M, Sinclair I, Hinton D, Boardman R, Earl G. The use of computed tomography for the study of archaeological coins. J Archaeol Sci Rep. 2016;6:3541. DOI: 10.1016/j.jasrep.2016.01.019.

13. Sanger MC. Investigating pottery vessel manufacturing techniques using radiographic imaging and computed tomography: Studies from the Late Archaic American Southeast. J Archaeol Sci Rep. 2016;9:586-98. DOI: 10.1016/j.jasrep.2016.08.005. 
14. Uldin T. Virtual anthropology - a brief review of the literature and history of computed tomography. Forensic Sciences Research. 2017;2(4):165-73. DOI:

10.1080/20961790.2017.1369621.

15. Franchi A, Valette S, Agier R, Prost R, Kechichan R, Fanton L. The prospects for application of computational anatomy in forensic anthropology for sex determination. Forensic Sci Int. 2019;297:156-60. DOI: 10.1016/j.forsciint.2019.01.009.

16. University of New Mexico. The New Mexico Decedent Image Database 2020 [updated 2020]. Accessed: 27th May 2020. Available from: https://nmdid.unm.edu/.

17. Dirkmaat DC, Cabo LL. Forensic Anthropology: Embracing the New Paradigm. In: Dirkmaat DC, editor. A Companion to Forensic Anthropology: Blackwell Publishing Ltd; 2012. p. 1-40.

18. Michel J, Paganelli A, Varoquaux A, Piercecchi-Marti MD, Adalian P, Leonetti G, et al. Determination of sex: interest of frontal sinus 3D reconstructions. J Forensic Sci. 2015;60(2):269-73. DOI: 10.1111/1556-4029.12630.

19. Franklin D, Swift L, Flavel A. 'Virtual anthropology' and radiographic imaging in the Forensic Medical Sciences. Egypt J Forensic Sci. 2016;6(2):31-43. DOI:

10.1016/j.ejfs.2016.05.011.

20. Guyomarc'h P, Santos F, Dutailly B, Desbarats P, Bou C, Coqueugniot H. Threedimensional computer-assisted craniometrics: a comparison of the uncertainty in measurement induced by surface reconstruction performed by two computer programs. Forensic Sci Int. 2012;219(1-3):221-7. DOI: 10.1016/j.forsciint.2012.01.008.

21. Ford JM, Decker SJ. Computed tomography slice thickness and its effects on threedimensional reconstruction of anatomical structures. J Forensic Radiol Imaging. 2016;4:436. DOI: 10.1016/j.jofri.2015.10.004.

22. Carew RM, Morgan RM, Rando C. A Preliminary Investigation into the Accuracy of 3D Modeling and 3D Printing in Forensic Anthropology Evidence Reconstruction. J Forensic Sci. 2019;64(2):342-52. DOI: 10.1111/1556-4029.13917.

23. Conlogue $\mathrm{G}$. Considered limitations and possible applications of computed tomography in mummy research. Anat Rec (Hoboken). 2015;298(6):1088-98. DOI: 10.1002/ar.23151.

24. Errickson D, Grueso I, Griffith SJ, Setchell JM, Thompson TJU, Thompson CEL, et al. Towards a Best Practice for the Use of Active Non-contact Surface Scanning to Record Human Skeletal Remains from Archaeological Contexts. Int J Osteoarchaeol. 2017;27(4):650-61. DOI: 10.1002/oa.2587.

25. Brough AL, Morgan B, Robinson C, Black S, Cunningham C, Adams C, et al. A minimum data set approach to post-mortem computed tomography reporting for anthropological biological profiling. Forensic Sci Med Pathol. 2014;10(4):504-12. DOI: 10.1007/s12024-014-9581-4.

26. Hodgdon T, Danrad R, Patel MJ, Smith SE, Richardson ML, Ballard DH, et al. Logistics of Three-dimensional Printing: Primer for Radiologists. Acad Radiol. 2018;25(1):4051. DOI: 10.1016/j.acra.2017.08.003.

27. Ballard DH, Trace AP, Ali S, Hodgdon T, Zygmont ME, DeBenedectis CM, et al. Clinical Applications of 3D Printing: Primer for Radiologists. Acad Radiol. 2018;25(1):52-65. DOI: 10.1016/j.acra.2017.08.004.

28. Archaeology Data Service., Digital Antiquity. Guides to Good Practice 2009 [updated 2009]. Accessed: 17th January 2019. Available from:

http://guides.archaeologydataservice.ac.uk/g2gpwiki.

29. Fedorov A, Beichel R, Kalpathy-Cramer J, Finet J, Fillion-Robin JC, Pujol S, et al. 3D Slicer as an image computing platform for the Quantitative Imaging Network. Magn Reson Imaging. 2012;30(9):1323-41. DOI: 10.1016/j.mri.2012.05.001.

30. Cheng GZ, San Jose Estepar R, Folch E, Onieva J, Gangadharan S, Majid A. Threedimensional Printing and 3D Slicer: Powerful Tools in Understanding and Treating Structural Lung Disease. Chest. 2016;149(5):1136-42. DOI: 10.1016/j.chest.2016.03.001. 
31. Marquez-Grant N. An overview of age estimation in forensic anthropology: perspectives and practical considerations. Ann Hum Biol. 2015;42(4):308-22. DOI: 10.3109/03014460.2015.1048288.

32. Minnema J, van Eijnatten M, Hendriksen AA, Liberton N, Pelt DM, Batenburg KJ, et al. Segmentation of dental cone-beam CT scans affected by metal artifacts using a mixedscale dense convolutional neural network. Med Phys. 2019. DOI: 10.1002/mp.13793. 33. CloudCompare. CloudCompare Version 2.6.1 User manual 2015 [updated 22nd February 2015]. Accessed: 4th May 2020. Available from:

https://www.cloudcompare.org/doc/qCC/CloudCompare\%20v2.6.1\%20\%20User\%20manual.pdf.

34. Villa C, Flies MJ, Jacobsen C. Forensic 3D documentation of bodies: Simple and fast procedure for combining CT scanning with external photogrammetry data. J Forensic Radiol Imaging. 2018;12:e2-e7. DOI: 10.1016/j.jofri.2017.11.003.

35. Carew RM, Morgan RM, Rando C. Experimental assessment of the surface quality of 3D printed bones. Aust J Forensic Sci. 2020. DOI: 10.1080/00450618.2020.1759684.

36. Brough AL, Bennett J, Morgan B, Black S, Rutty GN. Anthropological measurement of the juvenile clavicle using multi-detector computed tomography--affirming reliability. $J$ Forensic Sci. 2013;58(4):946-51. DOI: 10.1111/1556-4029.12126.

37. Carew RM, Viner MD, Conlogue G, Márquez-Grant N, Beckett S. Accuracy of computed radiography in osteometry: a comparison of digital imaging techniques and the effect of magnification. Journal of Forensic Radiology and Imaging. 2019:100348. DOI: 10.1016/j.jofri.2019.100348. 